\title{
On the Essential Norm of Multiplications Operators Acting on Cesàro Sequence Spaces
}

\author{
Julio C. Ramos-Fernández $\mathbb{D}^{1},{ }^{1}$ María A. Rivera-Sarmiento, ${ }^{1}$ and Margot Salas-Brown ${ }^{2}$ \\ ${ }^{1}$ Proyecto Curricular de Matemáticas, Facultad de Ciencias y Educación, Universidad Distrital Francisco José de Caldas, \\ Carrera 3 No. 26A-40, Bogotá, Colombia \\ ${ }^{2}$ Departamento de Matemáticas Aplicadas y Ciencias de la Computación, Universidad del Rosario, \\ Calle 12C No. 6-25, Bogotá, Colombia \\ Correspondence should be addressed to Julio C. Ramos-Fernández; julio.ramos.fernandez@gmail.com
}

Received 11 December 2018; Accepted 7 February 2019; Published 3 March 2019

Academic Editor: Raúl E. Curto

Copyright (C) 2019 Julio C. Ramos-Fernández et al. This is an open access article distributed under the Creative Commons Attribution License, which permits unrestricted use, distribution, and reproduction in any medium, provided the original work is properly cited.

In this article, we establish an important property about the growth of sequences in the dual space of Cesàro sequence spaces. As a consequence of this fact, we calculate the measure of noncompactness or the essential norm of the multiplication operator $M_{u}$ acting on Cesàro sequence spaces ces .

\section{Introduction}

During the past decade, there has been increasing interest in properties of multipliers between functional Banach spaces of real or complex sequences. Given two Banach spaces $X$ and $Y$, whose elements are sequences of real or complex numbers, a multiplier for $X$ and $Y$ is defined to be a numeric sequence (real or complex) $u=\{u(n)\}$ such that $u \cdot f \in Y$ for all $f \in$ $X$, where - denotes pointwise function multiplication and is dropped henceforward, with no confusion resulting; that is, $u f$ is the sequence $\{u(n) f(n)\}$. Each multiplier $u=\{u(n)\}$ induces a linear operator $M_{u}: X \longrightarrow Y$ given by $M_{u}(f)=$ $u \cdot f$. In case $M_{u}$ is continuous, we call it the multiplication operator from $X$ to $Y$ with symbol $u$.

Multiplication operators are classical and continue to be widely studied. In particular, multipliers of spaces of measurable functions were thoroughly examined during the mid-twentieth century. For example, Halmos's monograph [1, Ch. 7] contains important information about multiplication operators on the Hilbert space $L_{2}(\mu)$ of square integrable measurable functions with respect to a given measure $\mu$.

We also mention here the pioneering work Singh/Kumar in $[2,3]$ on properties of multiplication operators acting on spaces of measurable functions. These authors studied the compactness and closedness of the range of multiplication operators on $L_{2}(\mu)$. Additionally, we note that the work of Arora et al. in [4-6] examined properties of $M_{u}$ on Lorentz and Lorentz-Bochner spaces. Further significant results regarding multiplication operators were obtained by Castillo et al. in [7-9], in which these authors showed that the techniques used by previously mentioned authors can be modified to study multiplication operators on weak $L_{p}$ spaces, Orlicz-Lorentz spaces, and variable Lebesgue spaces.

Motivated by the idea of finding nonnull compact multiplication operators, recently, interesting works about properties of multiplication operators acting on Banach sequences spaces have appeared. Arora et al. in [6] show that a multiplication operator $M_{u}$ acting on Lorentz sequence spaces is compact if and only if $u \in c_{0}$, that is, if and only if $u(n) \longrightarrow 0$ as $n \longrightarrow \infty$. This last condition also characterizes the compactness of this operator $M_{u}$, acting on other Banach sequence spaces such as Orlicz-Lorentz sequence spaces [10], Cesàro sequence spaces [11], CesàroOrlicz sequence spaces [12], among others. However, the above spaces are classified as Köthe sequence spaces and the characterization of compactness (and other properties) of multiplication operators acting on Köthe sequence spaces is due to Ramos-Fernández and Salas-Brown [13] (see also $[14,15])$. It is an open problem to characterize the multipliers $u$ between two Köthe spaces $X$ and $Y$. 
Related to the problem of the characterization of the compactness of multiplication operators we have the problem of estimating its essential norm or the noncompactness measure of multiplication operators. This subject has been widely studied in the context of analytic functions. In the case of multiplication operators $M_{u}$ acting on nonatomic Köthe spaces the essential norm was calculate by Castillo et al. [15] and it is an open problem calculates the essential norm of this operator in a more general sense. The essential norm of multiplication operators acting on Lorentz sequence spaces was calculated by Castillo et al. [16]. The main objective of this article is to calculate the essential norm of multiplication operators acting on Cesàro sequence spaces. More precisely, we are going to show the following result.

Main Theorem. Suppose that $p>1$ and let $u=\{u(n)\}$ be a bounded sequence. Then for the operator $M_{u}: c e s_{p} \longrightarrow c e s_{p}$, we have

$$
\left\|M_{u}\right\|_{e}=\limsup _{n \rightarrow \infty}|u(n)|
$$

We present the proof of the above result in Section 3, and in Section 2 we gather some properties of the Cesàro sequence spaces and its dual given a new property for sequences in this space.

\section{Some Remarks of Cesàro Sequence Spaces}

The Cesàro sequence spaces appeared in 1968 in connection with the problem of the Dutch Mathematical Society to find their duals. This sequence space was first defined by Shiue [17] in 1970 as the set ces $p$ of all real sequences $x=\{x(n)\}$ such that

$$
\|x\|_{\text {ees }_{p}}:=\left(\sum_{n=1}^{\infty}\left(\frac{1}{n} \sum_{k=1}^{n}|x(k)|\right)^{p}\right)^{1 / p}<\infty,
$$

where $p$ is a parameter fixed greater or equal than 1 ; in fact, Leibowitz [18] and Jagers [19] proved that $c e s_{1}=\{0\}$, ces $s_{p}$ are separable reflexive Banach spaces for $1<p<\infty$. The space ces $_{p}$ arises in a natural way from Hardy's inequality,

$$
\sum_{n=1}^{\infty}\left(\frac{1}{n} \sum_{k=1}^{n}|x(k)|\right)^{p} \leq\left(\frac{p}{p-1}\right)^{p} \sum_{k=1}^{\infty}|x(k)|^{p}
$$

which establish that the $l_{p}$ spaces are continuously and strictly embedded into ces $_{p}$ for $1<p<\infty$.

For $k \in \mathbb{N}$ fixed, the sequence $e_{k}=\left\{e_{k}(n)\right\}$ defined by

$$
e_{k}(n)= \begin{cases}1, & n=k \\ 0, & \text { otherwise }\end{cases}
$$

is an element of $c e s_{p}$ and, in this case, we have

$$
\left\|e_{k}\right\|_{\operatorname{ces}_{p}}^{p}=\sum_{n=k}^{\infty} \frac{1}{n^{p}} .
$$

Observe that $\left\|e_{k}\right\|_{c e s_{p}} \longrightarrow 0$ as $k \longrightarrow \infty$. Furthermore, the sequence of canonical vectors $\left(e_{k}\right)_{k=1}^{\infty}$ is an unconditional, boundedly complete, and shrinking Schauder basis for $c e s_{p}$ (see [20]). Also, it is important to remark the following property which tell us that ces ${ }_{p}$ is a solid space. More precisely, if $x=\{x(n)\}$ and $y=\{y(n)\}$ are real sequences such that $y \in \operatorname{ces}_{p}$ with $1<p<\infty$ and $|x(n)| \leq|y(n)|$ for all $n \in \mathbb{N}$, then $x \in$ ces $_{p}$ and $\|x\|_{\text {ces }_{p}} \leq\|y\|_{\text {ces }_{p}}$.

The problem of the dual space of ces $_{p}$ was solved by Jagers in [19]. He showed that $\operatorname{ces}_{p}^{*}$ can be identified with its Köthe dual of all the sequences $y=\{y(n)\}$ such that

$$
\sum_{n=1}^{\infty}|y(n) x(n)|<\infty
$$

for all real sequence $x=\{x(n)\} \in$ ces $_{p}$. In particular, this means that for each bounded linear functional $F \in \operatorname{ces}_{p}^{*}$ we can find a real sequence $y \in c_{0}$ such that

$$
F(x)=\sum_{n=1}^{\infty} y(n) x(n)
$$

for all $x \in \operatorname{ces}_{p}$. We refer this fact as the representation Riesz's theorem for the Cesàso spaces. The description of the dual of $c e s_{p}$ given by Jagers in [19] can be quite complicated; it is given in terms of the least decreasing majorant; namely,

$$
\begin{aligned}
\operatorname{ces}_{p}^{*} & =\left\{x=\{x(n)\}:\|x\|_{\operatorname{ces}_{p}^{*}}\right. \\
: & \left.=\left(\sum_{n=1}^{\infty} n(\tilde{x}(n)-\tilde{x}(n+1))^{q}\right)^{1 / q}<\infty\right\},
\end{aligned}
$$

where $1 / p+1 / q=1$ and $\tilde{x}$ is the least decreasing majorant of $|x|$, with the property that, for $n>m$,

$$
(\tilde{x}(m)-\tilde{x}(n))\left(\sum_{k=m}^{n-1} \frac{1}{k^{p}}\right)^{-1}
$$

increases with $n$ if $m$ is fixed. However, Bennett [21] gave a simpler, though just isomorphic, identification of the dual space ces $_{p}^{*}$. For $1<p<\infty$, Bennet considers the Banach space $d(p)$ of all real sequence $x=\{x(n)\}$ such that

$$
\|x\|_{d(p)}:=\left(\sum_{n=1}^{\infty}\left(\sup _{k \geq n}|x(k)|^{p}\right)\right)^{1 / p}<\infty .
$$

Observe that if $x \in d(p)$, then $|x(n)| \longrightarrow 0$ as $n \longrightarrow \infty$ which implies that $d(p)$ is contained in $c_{0}$. Then for $1<p<\infty$, the dual space $c e s_{p}^{*}$ of $c e s_{p}$ can be identified with the sequence space $d(q)$, where $1 / p+1 / q=1$. Furthermore, for all $x \in$ ces $_{p}^{*}$ Bennett shows that

$$
\frac{1}{q}\|x\|_{d(q)} \leq\|x\|_{\text {ees }_{p}^{*}} \leq(p-1)^{1 / p}\|x\|_{d(q)} .
$$

We finish this section establishing the following property of the sequences in the space $d(q)$ which will be of great utility for the proof of the main result of this article. A similar result for sequences in ces $_{p}$ appears in [20]. 
Proposition 1. Suppose that $1<q<\infty$. If $x=\{x(n)\} \in d(q)$ then

$$
\lim _{n \longrightarrow \infty} n^{1 / q}|x(n)|=0 .
$$

Proof. It is enough to see that $d_{n}=n|x(n)|^{q} \longrightarrow 0$ as $n \longrightarrow \infty$. For any $n \in \mathbb{N}$ and $m \in \mathbb{N}$ such that $m \geq n$ we have

$$
\begin{aligned}
\sum_{k=n}^{\infty}\left(\sup _{j \geq k}|x(j)|^{q}\right) & \geq \sum_{k=n}^{m}\left(\sup _{j \geq k}|x(j)|^{q}\right) \\
& \geq(m-n+1)|x(m)|^{q} .
\end{aligned}
$$

In particular, for $m=2 n$, we have

$$
\begin{aligned}
d_{2 n} & =(2 n)|x(2 n)|^{q} \\
& \leq 2 \sum_{k=n}^{\infty}\left(\sup _{j \geq k}|x(j)|^{q}\right)+2|x(2 n)|^{q} \longrightarrow 0
\end{aligned}
$$

as $n \longrightarrow \infty$ since $x \in d(q)$ and $|x(n)| \longrightarrow 0$ as $n \longrightarrow \infty$. While, for $m=2 n-1$, we have

$$
\begin{aligned}
d_{2 n-1} & =(2 n-1)|x(2 n-1)|^{q} \\
& \leq 2 \sum_{k=n}^{\infty}\left(\sup _{j \geq k}|x(j)|^{q}\right)-|x(2 n-1)|^{q} \longrightarrow 0
\end{aligned}
$$

as $n \longrightarrow \infty$. This shows the result.

Remark 2. The set of all sequences $x=\{x(n)\}$ satisfying the condition (12) is a Banach space with the norm

$$
\|x\|=\sup _{n \in \mathbb{N}} n^{1 / q}|x(n)| .
$$

More precisely, if $\beta=\{\beta(n)\}$ is a sequence of positive numbers, the set $l_{\beta}^{\infty}$ of all sequences $x=\{x(n)\}$ such that

$$
\|x\|_{\beta}=\sup _{n \in \mathbb{N}} \beta(n)|x(n)|<\infty
$$

is a Banach space which has a closed subspace $c_{0}^{\beta}$ of all sequences $x=\{x(n)\}$ such that

$$
\lim _{n \longrightarrow \infty} \beta(n)|x(n)|=0 .
$$

This kind of spaces can be called growth spaces of sequences and they are generalizations of the classical spaces $l^{\infty}$ and $c_{0}$ (obtained when $\beta(n)=1$ for all $n \in \mathbb{N}$ ). Hence, $d(q)$ is contained in the growth space $c_{0}^{\beta}$ with $\beta(n)=n^{1 / q}$ for all $n \in \mathbb{N}$.

\section{The Essential Norm of Multiplication Operators on Cesàro Sequence Spaces}

We recall that if $\left(X,\|\cdot\|_{X}\right)$ is a Banach space and $T: X \longrightarrow X$ is a continuous operator, then the measure of noncompactness (or essential norm) of $T$, denoted by $\|T\|_{e}$, is the distance of $T$ to the class of the compact operators on $X$; that is,

$$
\|T\|_{e}=\inf \{\|T-K\|: K: X
$$

$$
\longrightarrow X \text { is a linear compact operator }\}
$$

where $\|T\|$ denotes the operator norm of $T$, which is defined by $\|T\|=\sup \left\{\|T f\|_{X}:\|f\|_{X}=1\right\}$. It is known that an operator $T: X \longrightarrow X$ is compact if and only if $\|T\|_{e}=0$.

In the case of multiplication operators $M_{\mathcal{u}}$ acting on Cesàro sequence spaces, recently Komal et al. [11] (see also [22]) showed the following result.

Theorem 3 (see [11]). Suppose that $p>1$ and that $u=$ $\{u(n)\}$ is a bounded sequence. The multiplication operator $M_{u}$ : ces $_{p} \longrightarrow$ ces $_{p}$ is compact if and only if $u \in c_{0}(|u(n)| \longrightarrow 0$ as $n \stackrel{\infty}{\longrightarrow})$.

The condition that $u$ is a bounded sequence in the above theorem is necessary and sufficient for the fact that the operator $M_{u}:$ ces $s_{p} \longrightarrow c e s_{p}$ will be continuous or bounded. Our goal is to obtain an expression of the essential norm of $M_{u}:$ ces $_{p} \longrightarrow$ ces $_{p}$ which implies the above result; more precisely, in our research, the result we have found is the following.

Theorem 4. Suppose that $p>1$ and let $u=\{u(n)\}$ be a bounded sequence. Then for the multiplication operator $M_{u}$ : ces $_{p} \longrightarrow$ ces $_{p}$ we have

$$
\left\|M_{u}\right\|_{e}=\limsup _{n \rightarrow \infty}|u(n)| .
$$

Proof. For each $N \in \mathbb{N}$, we set the sequence $u_{N}=(u(1), u(2)$, $\ldots, u(N), 0,0, \ldots)$. Then by Theorem 3 , the multiplication operator $M_{u_{N}}: c e s_{p} \longrightarrow c e s_{p}$ is compact. Hence

$$
\left\|M_{u}\right\|_{e} \leq\left\|M_{u}-M_{u_{N}}\right\|=\left\|M_{u-u_{N}}\right\| .
$$

But if $a=\{a(n)\} \in c e s_{p}$ is such that $\|a\|_{\text {ces }_{p}}=1$, then clearly

$$
\left|\left(u(n)-u_{N}(n)\right) \cdot a(n)\right| \leq S_{N}|a(n)|
$$

for all $n \in \mathbb{N}$, where $S_{N}=\sup \{|u(k)|: k \geq N\}$. Thus, since the space $c e s_{p}$ is solid, we conclude that the sequence $\left(u-u_{N}\right) \cdot a$ belongs to ces $_{p}$ and

$$
\begin{aligned}
\left\|M_{u}-M_{u_{N}}\right\| & \leq\left\|M_{u}(a)-M_{u_{N}}(a)\right\|_{\text {ces }_{p}} \\
& =\left\|\left(u-u_{N}\right) a\right\|_{\text {ces }_{p}} \leq S_{N}\|a\|_{\text {ces }_{p}}=S_{N} .
\end{aligned}
$$

Therefore $\left\|M_{u}\right\|_{e} \leq S_{N}$ for all $N \in \mathbb{N}$ and hence

$$
\left\|M_{u}\right\|_{e} \leq \limsup _{n \rightarrow \infty}|u(n)| \text {. }
$$

On the other hand, if $K:$ ces $_{p} \longrightarrow c e s_{p}$ is any compact operator, then, for $k \in \mathbb{N}$ fixed, we consider the sequence $e_{k}$ given by

$$
e_{k}(n)= \begin{cases}1, & n=k \\ 0, & \text { otherwise }\end{cases}
$$


We have

$$
\left\|e_{k}\right\|_{\text {ces }_{p}}^{p}=\sum_{n=k}^{\infty} \frac{1}{n^{p}} \geq \int_{k}^{\infty} \frac{d x}{x^{p}}=\frac{1}{p-1} k^{1-p}
$$

and hence $e_{k} \in$ ces $s_{p}$ for all $k \in \mathbb{N}$ and $\left\|e_{k}\right\|_{c e s_{p}} \longrightarrow 0$ as $k \longrightarrow$ $\infty$ since it is the tail of a convergent series. Furthermore, the sequence $\left\{f_{k}\right\} \subseteq$ ces $_{p}$ defined by $f_{k}=e_{k} /\left\|e_{k}\right\|_{c e s_{p}}$ satisfies that $\left\|f_{k}\right\|_{c e s_{p}}=1$ for all $k \in \mathbb{N}$, and, in particular, $\left\{f_{k}\right\}$ is a bounded sequence in $\operatorname{ces}_{p}$. We are going to show that

$$
\lim _{k \rightarrow \infty}\left\|K\left(f_{k}\right)\right\|_{\text {ces }_{p}}=0 .
$$

Indeed, if (27) is false, then by passing to a subsequence if is necessary and without loss of generality, we can suppose that there exists $\delta>0$ such that

$$
\left\|K\left(f_{k}\right)\right\|_{\text {ces }_{p}} \geq \delta
$$

for all $k \in \mathbb{N}$. Since $K:$ ces $_{p} \longrightarrow$ ces $p$ is a compact operator and $\left\{f_{k}\right\}$ is bounded in $c e s_{p}$, then by passing to a subsequence if is necessary, we can suppose that $\left\{K\left(f_{k}\right)\right\}$ converges in ces $p$. That is, there exists a $b \in c e s_{p}$ such that

$$
\lim _{k \rightarrow \infty}\left\|K\left(f_{k}\right)-b\right\|_{c e s_{p}}=0 .
$$

Note that, if $b=0$, then this fact leads us to contradict (28). By Hahn-Banach's theorem, it is enough to show that $h(b)=$ 0 for all bounded linear functionals $h$ on $c e s_{p}$. Let $h$ be any bounded linear functional on $c e s_{p}$. Then the composition $h K$ is also a bounded linear functional on $c e s_{p}$, so that, by the Riesz representation theorem for ces $s_{p}$ spaces, there exist $a=$ $\{a(n)\} \in c_{0}$ such that

$$
h K(c)=\sum_{n=1}^{\infty} a(n) \cdot c(n)
$$

for all $c=\{c(n)\} \in \operatorname{ces}_{p}$. In particular, evaluating at $f_{k}$, we have

$$
\left|h K\left(f_{k}\right)\right|=\frac{|a(k)|}{\left\|e_{k}\right\|_{c e s_{p}}} \leq k^{1 / q}|a(k)| \longrightarrow 0
$$

as $m \longrightarrow \infty$ in virtue of Proposition 1 . Hence

$$
\begin{aligned}
|h(b)| & \leq\left|h(b)-h K\left(f_{k}\right)\right|+\left|h K\left(f_{k}\right)\right| \\
& \leq\|h\|\left\|b-K\left(f_{k}\right)\right\|+\frac{|a(k)|}{\left\|e_{k}\right\|_{\text {ces }_{p}}} \longrightarrow 0
\end{aligned}
$$

as $m \longrightarrow \infty$ and $h(b)=0$. This proves the claim. Next we can conclude the proof of our result. Since $f_{k}$ is a unitary vector in $c e s_{p}$ for each $k \in \mathbb{N}$, we can write

$$
\begin{aligned}
\left\|M_{u}-K\right\| & \geq\left\|M_{u}\left(f_{k}\right)-K\left(f_{k}\right)\right\|_{\text {ces }_{p}} \\
& \geq \frac{1}{\left\|e_{k}\right\|}\left\|M_{u}\left(e_{k}\right)\right\|_{\text {ces }_{p}}-\left\|K\left(f_{k}\right)\right\|_{\text {ces }_{p}} \\
& =\frac{1}{\left\|e_{k}\right\|}\left\|u \cdot e_{k}\right\|_{c e s_{p}}-\left\|K\left(f_{k}\right)\right\|_{c e s_{p}} \\
& =|u(k)|-\left\|K\left(f_{k}\right)\right\|_{\text {ces }_{p}} .
\end{aligned}
$$

Thus taking the limit when $k \longrightarrow \infty$, we conclude that

$$
\left\|M_{u}-K\right\| \geq \limsup _{k \rightarrow \infty}|u(k)|
$$

and therefore $\left\|M_{u}\right\|_{e} \geq \lim \sup _{n \rightarrow \infty}|u(n)|$ since the compact operator $K$ on $c e s_{p}$ was arbitrary. This finishes the proof.

Remark 5. We finish this article with the following comment: the fact that the result obtained in this article is also valid for the other Banach spaces of sequences as, for example, Lorentz sequence spaces such as that we can see in [16], is very interesting. Lorentz sequence spaces and Cesàro sequence spaces are examples of Köthe sequence spaces. A Banach space of sequences $X$ is said to be a Köthe sequence space if it satisfies the following:

(i) If $a, b$ are sequences such that $|a(k)| \leq|b(k)|$ for all $k \in \mathbb{N}$ and $b \in X$, then $a \in X$ and $\|a\|_{X} \leq\|b\|_{X}$.

(ii) If $A=\left\{n_{1}, n_{2}, \ldots, n_{m}\right\}$ is a finite subset of $\mathbb{N}$, then the sequence $\mathbf{1}_{A}$, defined by

$$
\mathbf{1}_{A}(k)= \begin{cases}1, & k \in A, \\ 0, & k \notin A,\end{cases}
$$

belongs to $X$.

Thus, it is natural to ask if the result obtained in this article (and in [16]) is also valid for multiplication operator acting on any Köthe sequence space $X$.

\section{Data Availability}

No data were used to support this study.

\section{Conflicts of Interest}

The authors declare that they have no conflicts of interest regarding the publication of this paper.

\section{References}

[1] P. R. Halmos, A Hilbert Space Problem Book, Van Nostrand, Princeton, NJ, USA, 1967.

[2] R. K. Singh and A. Kumar, "Multiplication operators and composition operators with closed ranges," Bulletin of the Australian Mathematical Society, vol. 16, no. 2, pp. 247-252, 1977.

[3] R. K. Singh and A. Kumar, "Compact composition operators," Journal of the Australian Mathematical Society: Pure Mathematics and Statistics. Series A, vol. 28, no. 3, pp. 309-314, 1979.

[4] S. C. Arora, G. Datt, and S. Verma, "Multiplication operators on Lorentz spaces," Indian Journal of Mathematics, vol. 48, no. 3, pp. 317-329, 2006.

[5] S. C. Arora, G. Datt, and S. Verma, "Multiplication and composition operators on Lorentz-Bochner spaces," Osaka Journal of Mathematics, vol. 45, no. 3, pp. 629-641, 2008.

[6] S. C. Arora, G. Datt, and S. Verma, "Operators on Lorentz sequences spaces," Academy of Sciences of the Czech Republic. Mathematical Institute. Mathematica Bohemica, vol. 134, no. 1, pp. 87-98, 2009. 
[7] R. E. Castillo, H. C. Chaparro, and J. C. Ramos-Fernández, "Orlicz-Lorentz spaces and their multiplication operators," Hacettepe Journal of Mathematics and Statistics, vol. 44, no. 5, pp. 991-1009, 2015.

[8] R. E. Castillo, J. C. Ramos-Fernández, and H. Rafeiro, "Multiplication operators in variable Lebesgue spaces," Revista Colombiana de Matemáticas, vol. 49, no. 2, pp. 293-305, 2015.

[9] R. E. Castillo, F. A. Vallejo Narvaez, and J. C. Ramos-Fernández, "Multiplication and composition operators on weak $L_{p}$ spaces," Bulletin of the Malaysian Mathematical Sciences Society, vol. 38, no. 3, pp. 927-973, 2015.

[10] P. Bala, A. Gupta, and N. Bhatia, "Multiplication operators on Orlicz-Lorentz sequence spaces," International Journal of Mathematical Analysis, vol. 7, no. 29-32, pp. 1461-1469, 2013.

[11] B. S. Komal, S. Pandoh, and K. Raj, "Multiplication operators on Cesàro sequence spaces," Demonstratio Mathematica, vol. 49, no. 4, pp. 430-436, 2016.

[12] K. Raj, C. Sharma, and S. Pandoh, "Multiplication operators on Cesàro-Orlicz sequence spaces," Fasciculi Mathematici, no. 57, pp. 137-145, 2016.

[13] J. C. Ramos-Fernández and M. Salas-Brown, "On multiplication operators acting on Köthe sequence spaces," Afrika Matematika, vol. 28, no. 3-4, pp. 661-667, 2017.

[14] H. Hudzik, R. Kumar, and R. Kumar, "Matrix multiplication operators on Banach function spaces," The Proceedings of the Indian Academy of Sciences - Mathematical Sciences, vol. 116, no. 1, pp. 71-81, 2006.

[15] R. E. Castillo, H. Rafeiro, J. C. Ramos-Fernández, and M. SalasBrown, "Multiplication operator on köthe spaces: measure of non-compactness and closed range," Bulletin of the Malaysian Mathematical Sciences Society, pp. 1-12, 2017.

[16] R. E. Castillo, J. C. Ramos-Fernández, and M. Salas-Brown, "The essential norm of multiplication operators on Lorentz sequence spaces," Real Analysis Exchange, vol. 41, no. 1, pp. 245251, 2016.

[17] J. S. Shiue, "On the Cesáro sequence space," Tamkang Journal of Mathematics, vol. 1, no. 1, pp. 19-25, 1970.

[18] G. M. Leibowitz, "A note on the Cesàro sequence spaces," Tamkang Journal of Mathematics, vol. 2, no. 2, pp. 151-157, 1971.

[19] A. A. Jagers, "A note on Cesáro sequence spaces," Nieuw Archief voor Wiskunde, vol. 22, no. 3, pp. 113-124, 1974.

[20] G. P. Curbera and W. J. Ricker, "Solid Extensions of the Cesàro Operator on $\ell^{p}$ and $c_{0}$," Integral Equations and Operator Theory, vol. 80, no. 1, pp. 61-77, 2014.

[21] G. Bennett, Factorizing the Classical Inequalities, vol. 120 of Memoirs of the American Mathematical Society, 1996.

[22] A. A. Albanese, J. Bonet, and W. J. Ricker, "Multiplier and averaging operators in the Banach spaces $\operatorname{ces}(\mathrm{p}), 1<\mathrm{p}<\infty$," Positivity, vol. 23, no. 1, pp. 177-193, 2018. 


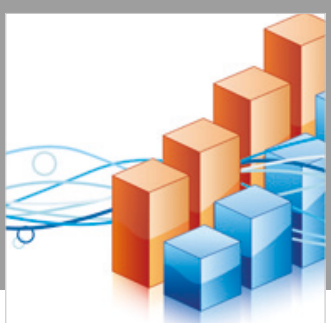

Advances in

Operations Research

\section{-n-m}
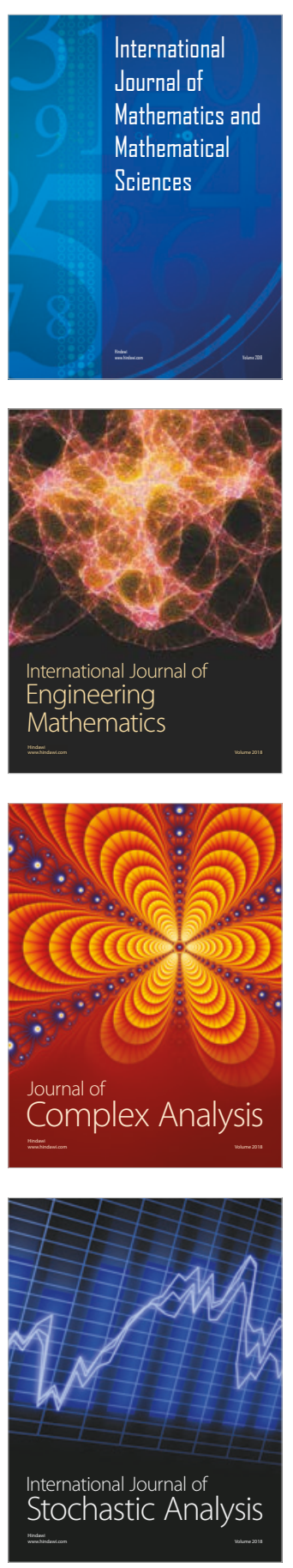
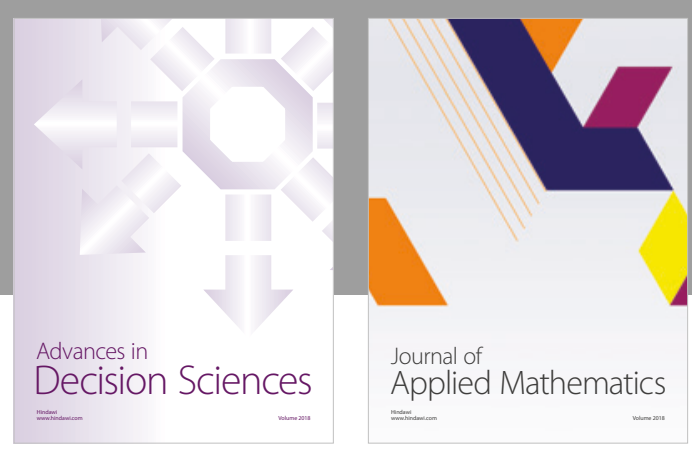

Journal of

Applied Mathematics
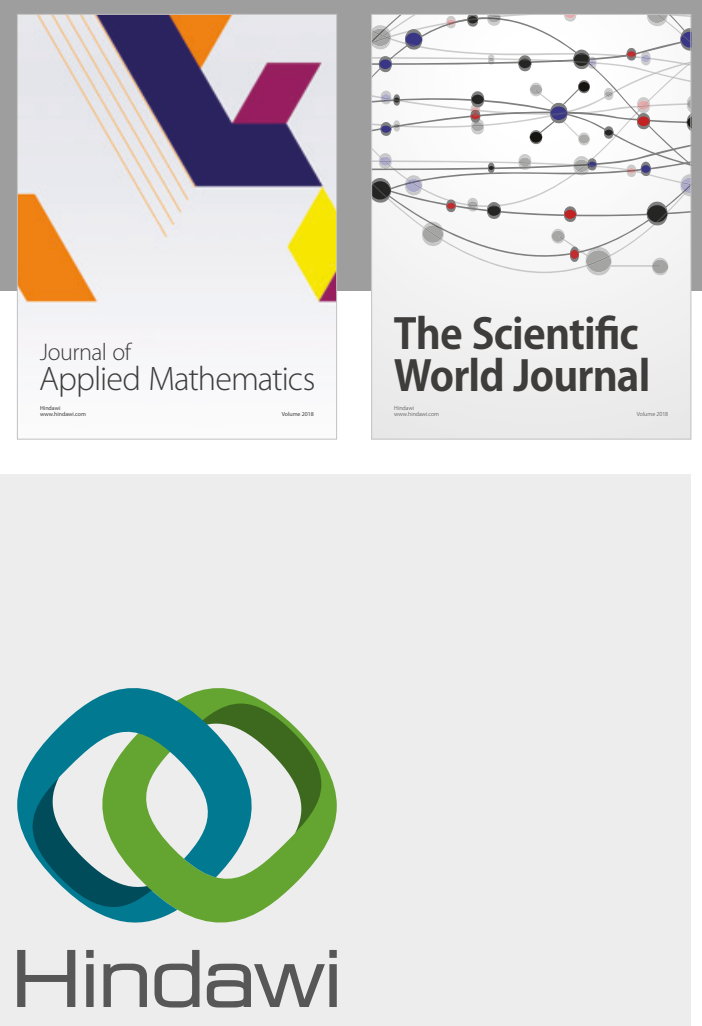

Submit your manuscripts at

www.hindawi.com

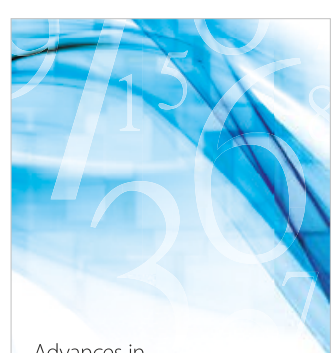

Advances in
Numerical Analysis
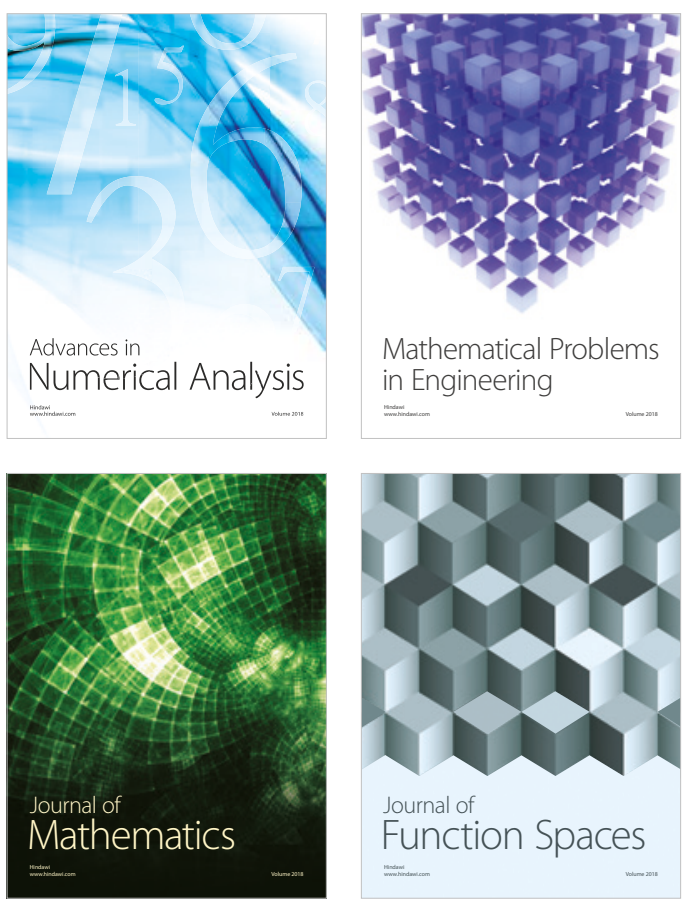

Mathematical Problems in Engineering

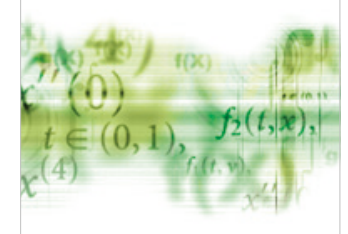

International Journal of

Differential Equations

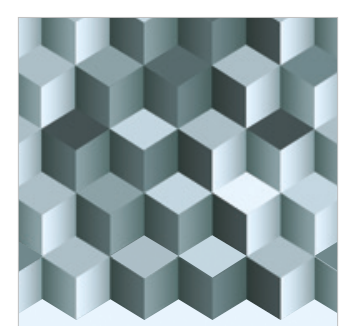

Journal of

Function Spaces

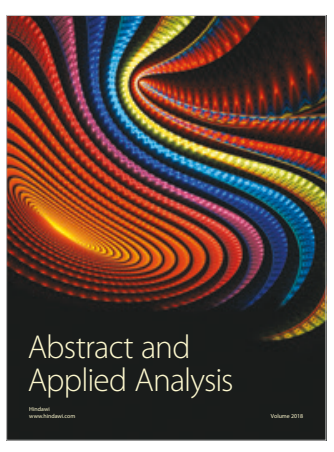

The Scientific

World Journal

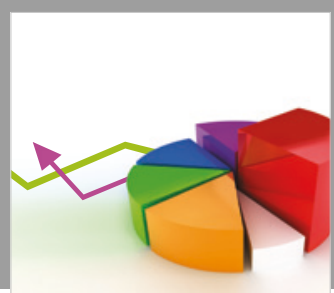

Journal of

Probability and Statistics
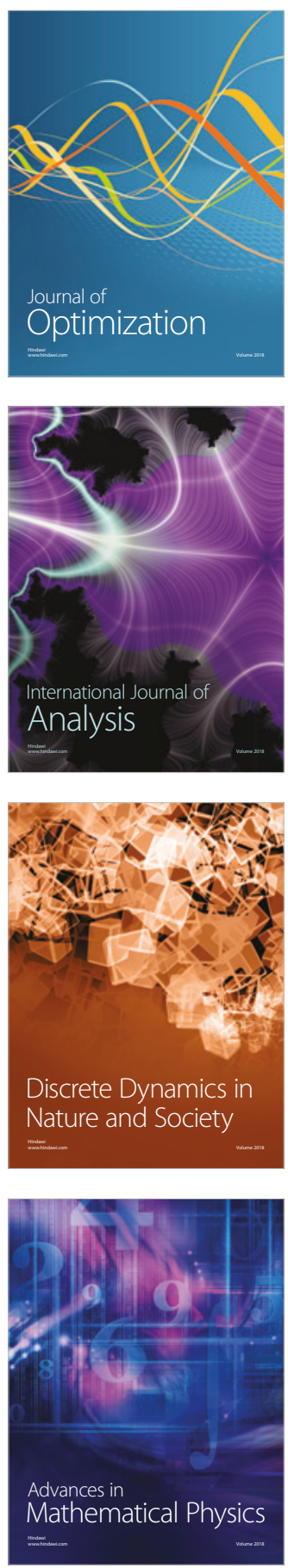